\title{
Erratum: Lifting mean-field degeneracies in anisotropic classical spin systems [Phys. Rev. B 92, 155131 (2015)]
}

\author{
Yuriy Sizyuk, Natalia B. Perkins, and Peter Wölfle \\ (Received 13 July 2016; published 2 August 2016)
}

DOI: 10.1103/PhysRevB.94.079901

In our code, we found an error that caused us to choose the wrong mean-field state as the ground state for $K>1$ (with $J=-1$ as in the rest of the paper). In this parameter range, the ground state is a set of planes stacked AFM along one of the cubic axes with ordering vectors $(\pi, 0,0),(0, \pi, 0)$, and $(0,0, \pi)$. The direction of moments in these states is chosen on the mean field level to be cubic $\pm \hat{x}, \pm \hat{y}$, or $\pm \hat{z}$ corresponding to the direction of the AFM stacking (see Fig. 1). This invalidates our result of intermediate phase with minima and maxima "dancing" around each other, including Figs. 2 and 3 (see the original paper), as well as the phase with moments along the body diagonals, however, the method presented in the paper remains valid and useful. The application of the method for $K<1$ is not affected by this problem. The corrected version of the paper can be found in the original paper.
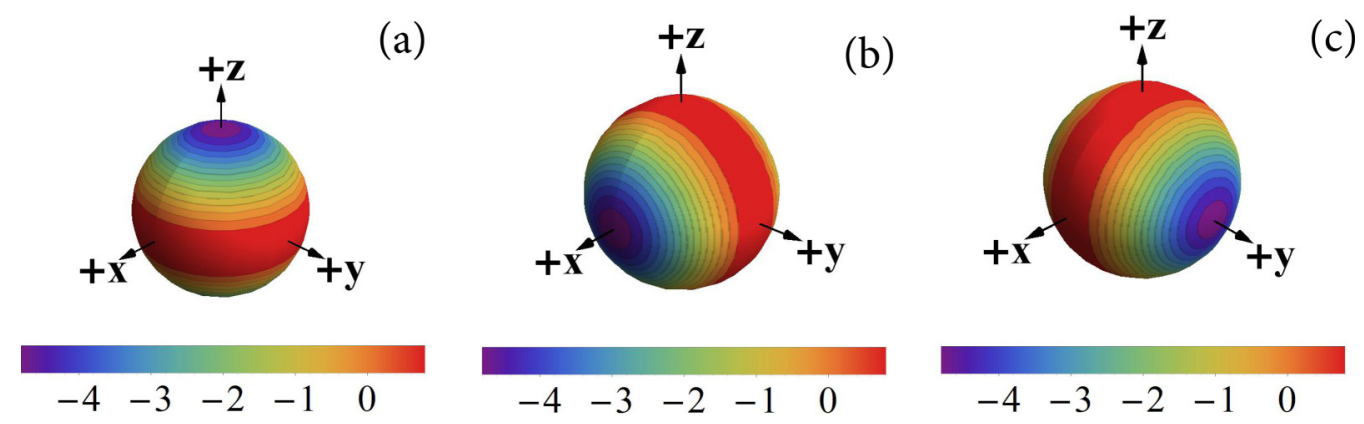

FIG. 1. Mean-field energy of the model Eq. (14) computed at $J=-1$ and $K=1.4$ is plotted on the surface of the unit sphere. The preferred directions of the magnetization, corresponding to the minimum of energy, are shown by deep blue color. (a) $(0,0, \pi)$ state with stacking along $z$ direction; (b) $(\pi, 0,0)$ state with stacking along $x$ direction; (b) $(0, \pi, 0)$ state with stacking along $y$ direction. 